\title{
Min-Max MSE-based Interference Alignment for Transceiver Designs in Cognitive Radio Networks
}

\author{
Ha Hoang $K H A^{1}$, Hung Quang $T A^{2}$ \\ ${ }^{1}$ Faculty of Electrical and Electronics Engineering, Ho Chi Minh City University of Technology, VNU-HCM, Vietnam \\ ${ }^{2}$ Faculty of Information Technology, Hanoi University, Vietnam \\ hhkha@hcmut.edu.vn,hta@hanu.edu.vn
}

Submitted August 8, 2016 / Accepted February 22, 2017

\begin{abstract}
This paper is concerned with an optimal design of the precoders and receive filters for cognitive radio $(C R)$ networks in which multiple secondary users (SUs) share the same frequency band with multiple primary users (PUs). To cope with interference and to achieve fairness among users, we develop an interference alignment (IA) scheme by minimizing the maximum mean squared error (Min-Max MSE) of the received signals. Since the Min-Max MSE design problems are nonconvex in the design matrix variables of the precoders and receive filters, we develop an alternating optimization algorithm with provable convergence to iteratively find the optimal solutions. In each iteration, the precoder design problems can be recast as second order cone program (SOCP) while the optimal receive filters can be derived in closed-form solutions. Finally, numerical results are provided to demonstrate the superiority of the proposed method as compared to previous work in terms of the information rate and bit error rate.
\end{abstract}

\section{Keywords}

Multiuser MIMO, cognitive radio, interference alignment, Min-Max MSE, transceiver design

\section{Introduction}

With the growing demand of high data rate wireless services, radio frequency spectrum resources have recently become scare and precious. Thus, spectrum utilization efficiency has been of great concern in wireless communication network designs. Cognitive radio (CR) has been known as a wireless communication technique which renders radio spectrum exploitation more effective [1-4]. In CR networks, secondary users (SUs) can opportunistically access the spectrum licensed to primary users (PUs). However, the SUs are allowed to transmit their signals only if their transmission does not cause adverse interference to the PUs [5].

Of interest in this paper is a CR network in which $m u l$ tiple PUs and multiple SUs transmit at the same time in the same frequency band. In such a CR network, inter-user in- terference can be severe and significantly degrade the system performance. As the users are equipped with multiple antennas, additional spatial dimensions can be exploited to deal with interference. To deal with interference and improve the system performance in CR networks, the optimal transmission strategy designs for the SUs in the physical layer are crucial issues. Various performance metrics which are widely used for the transceiver designs in CR networks are the sum-rate and sum of mean square error (MSE) [5-8]. The authors in [6] designed the SU transmission strategies to maximize the SU weighted sum-rate while maintaining reliable communication for a single PU. Reference [7] designed the precoders and receive filters to maximize the sum-rate for cognitive MIMO ad-hoc networks with a single PU by using the weighted minimum MSE method. The authors in [9] developed a space alignment algorithm for a CR network with a single PU and single SU. Alternatively, reference [2] introduced the cooperative game theory for spectrum sharing in multiuser multiple-input multiple output (MIMO) cognitive radio networks.

Recently, interference alignment (IA) has known as an efficient approach to deal with interference in multiuser MIMO wireless networks [10-13]. The underlying idea of IA is to construct the transmitted signals such that the interference signals at each receiver are aligned into a reduced dimension subspace which is orthogonal to the one spanned by the desired signals [10-12]. IA has been applied into $K$-user interference channels [10], [12], wireless X networks [14], and heterogeneous networks (HetNets) [15]. Recently, it has been adapted and extended to CR networks, see [15-18] and references therein. In [18], the SU transmission is aligned into unused spatial directions of the PU channel. The authors in [15] applied an IA scheme for HetNets in which the macro-cell and small-cells operate in an underlay CR mode and the secondary terminals exploit unused space dimensions of the primary terminal for interference cancellation. Additionally, the authors in [19] developed IA techniques with different levels of network coordination for spectral coexistence of HetNets in terrestrial and satellite paradigms. Reference [20] employed IA for HetNets in which IA was developed for a quantized version of the alignment direction 
and joint IA and space-frequency block codes schemes were proposed. However, the models in almost all previous works are restricted to single primary user and multiple secondary users.

In this paper, we consider the optimal transceiver design for MIMO CR networks with multiple PUs and SUs. In order to efficiently mitigate interference and obtain fairness among users, we seek the transmission strategies to minimize the maximum user MSE (Min-Max MSE) among the PUs and SUs. To the best of our knowledge, the problem of Min-Max MSE based IA for interference mitigation in multiuser MIMO CR networks to achieve MSE fairness among users has not been considered yet. Since Min-Max MSE based design problems are highly nonconvex with respect to the matrix variables, the globally optimal solutions are difficult to obtain. This paper will propose the structures of the transceivers and iterative algorithms to obtain the suboptimal solutions. The main contributions of the paper are listed as follows:

- We formulate the transceiver designs of the PUs and SUs for the CR network as the Min-Max MSE IA problems to achieve fairness among the users in the same network. To guarantee the higher priority to the PUs, the PU transmission is designed without awareness of the presence of the SUs while the SUs must confine their transmitted signals into unused communication directions of the PU receivers to restrict interference to the PUs.

- We propose a structure of each SU transmit precoder as a cascade of two precoder matrices. The first matrix is selected to align the SU signals into a subspace orthogonal with a desired signal subspace at each PU receiver while the second matrix is designed to minimize the maximum user MSE with the individual transmit power constraint. To overcome difficulties associated with the nonconvexity of the design problems, we adopt the alternating optimization to decompose the design problems into tractable convex optimization subproblems. Specifically, we reformulate the precoder designs into the second-order cone programming (SOCP) problems which can be efficiently solved by interior-point methods. On the other hand, for the receive filter designs, we derive closed-form expressions for the optimal receive filters.

- We show the convergence of the proposed algorithm by theoretical analysis and numerical results. We also provide the computational complexity of the proposed algorithms.

- We provide numerical results to examine the performance of the proposed method in terms of user rates and BER in comparison with the IA scheme in [17] which uses the interference leakage minimization. The numerical results demonstrate the superior performance of the proposed method.

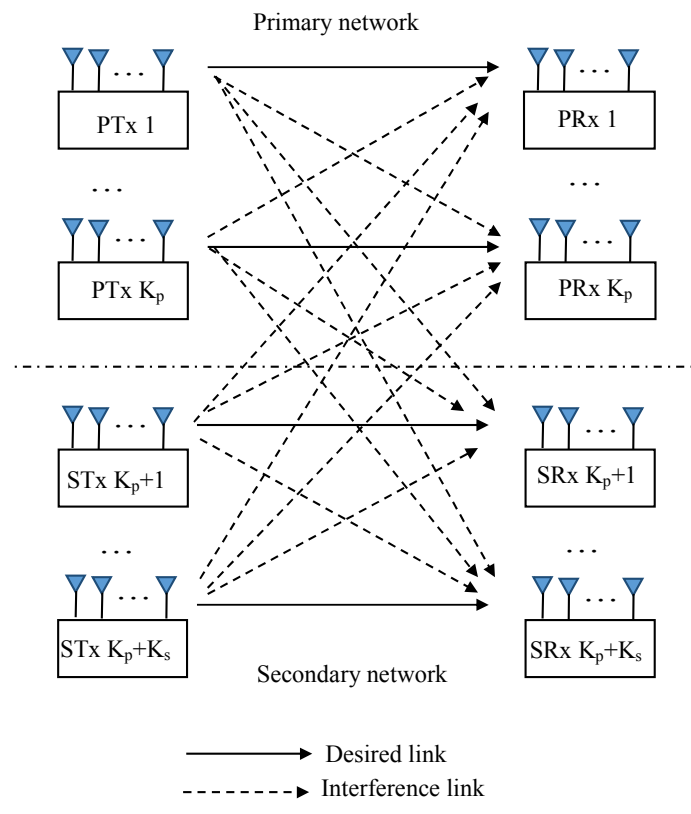

Fig. 1. A cognitive radio network with multiple PUs and SUs.

The rest of the paper is organized as follows. Section 2 describes the CR model considered in the paper. In Sec. 3, the transceiver design problems are formulated and the proposed method for the optimal transceiver designs is developed. Numerical results are provided in Sec. 4. Finally, Section 5 provides concluding remarks.

The following notations are used in the paper. Boldface upper (lower) case letters represent matrices (vectors). $\mathbf{X}^{H}$, $\mathbf{X}^{\dagger}, \operatorname{rank}(\mathbf{X}), \operatorname{tr}(\mathbf{X})$, and $\mathcal{N}(\mathbf{X})$ denote the Hermitian transposition, conjugate, rank, trace and null space of matrix $\mathbf{X}$. $[\mathbf{X}]_{k}^{\ell}$ is a matrix taking $\ell-k+1$ columns from column $k$ of matrix $\mathbf{X}$. $\operatorname{vec}(\mathbf{X})$ is the column vector obtained by stacking the columns of the matrix $\mathbf{X}$. $\|\cdot\|_{F}$ and $\|.\|_{2}$ are the Frobenius norm of a matrix and the norm-2 of a vector, respectively. $\otimes$ stands for the Kronecker product. $\operatorname{blkdiag}\left(\mathbf{X}_{1}, \mathbf{X}_{2}, \ldots, \mathbf{X}_{n}\right)$ is a block diagonal matrix in which the main diagonal blocks are $\mathbf{X}_{1}, \mathbf{X}_{2}, \ldots, \mathbf{X}_{n} . \mathbf{I}_{d}$ is a $d \times d$ identity matrix while $\mathbf{0}_{n \times m}$ is the $n \times m$ null matrix. A complex Gaussian random vector $\mathbf{x}$ with mean $\overline{\mathbf{x}}$ and covariance $\mathbf{R}_{\mathbf{z}}$ is represented by $\mathbf{x} \sim \mathcal{C N}\left(\overline{\mathbf{x}}, \mathbf{R}_{\mathbf{x}}\right)$.

\section{System Model}

Consider a wireless CR network in which $K_{s}$ pairs of the secondary transmitters (STxs) and secondary receivers (SRxs) share spectrum resources with $K_{p}$ pairs of the primary transmitters (PTxs) and primary receivers (PRxs) in an underlay method, as illustrated in Fig. 1. For ease of presentation, we define the set of PUs as $\mathcal{K}_{p}=\left\{1,2, \ldots K_{p}\right\}$ while that of SUs is denoted as $\mathcal{K}_{s}=\left\{K_{p}+1, K_{p}+2, \ldots, K_{p}+K_{s}\right\}$. The total number of users in the entire network is $K=K_{p}+K_{s}$ and the set of all the users in the network is $\mathcal{K}=\mathcal{K}_{p} \cup \mathcal{K}_{s}$. Assume that transmitter $k$ is equipped with $N_{k}$ antennas while receiver $k$ is equipped with $M_{k}$ antennas. Transmitter $k$ sends the signal $\mathbf{x}_{k} \in \mathbb{C}^{d_{k} \times 1}$, where $d_{k}$ is the number of data streams, to its associated receiver $k$. To be able to recover $d_{k}$ data streams 
at user $k$, it is required that $d_{k} \leq \min \left\{N_{k}, M_{k}\right\}$. We assume the transmitted symbols of $\mathbf{x}_{k}$ are independently Gaussian encoded symbols with $\mathbb{E}\left(\mathbf{x}_{k} \mathbf{x}_{k}^{H}\right)=\mathbf{I}$, and $\mathbb{E}\left(\mathbf{x}_{k} \mathbf{x}_{\ell}^{H}\right)=\mathbf{0}$ for $k \neq \ell$. To transmit $d_{k}$ data streams over $N_{k}$ antennas, transmitter $k$ performs a linear processing operation on the signal $\mathbf{x}_{k}$ by a linear precoder $\mathbf{F}_{k} \in \mathbb{C}^{N_{k} \times d_{k}}$. The transmitted power at user $k$ is constrained by

$$
\left\|\mathbf{F}_{k}\right\|_{F}^{2} \leq P_{k, \max }, \quad k \in \mathcal{K}
$$

where $P_{k \text {,max }}$ is the maximum transmit power at user $k$. The received signal at user $k^{1}$

$$
\mathbf{y}_{k}=\mathbf{H}_{k, k} \mathbf{F}_{k} \mathbf{x}_{k}+\sum_{\ell \in \mathcal{K} \backslash\{k\}} \mathbf{H}_{k, \ell} \mathbf{F}_{\ell} \mathbf{x}_{\ell}+\mathbf{z}_{k}, \quad k \in \mathcal{K}
$$

where $\mathbf{z}_{k} \in \mathbb{C}^{M_{k} \times 1}$ is an additive while Gaussian noise vector with $\mathbf{z}_{k} \sim \mathcal{C N}\left(\mathbf{0}, \sigma_{k}^{2} \mathbf{I}\right) . \mathbf{H}_{k, \ell} \in \mathbb{C}^{M_{k} \times N_{\ell}}$ is the channel matrix between transmitter $\ell$ and receiver $k$. The channels are assumed to be time-invariant over the transmission time period under consideration [2]. To retrieve $d_{k}$ data streams, receiver $k$ performs a linear signal processing operation on the received signal by a receive filter matrix $\mathbf{W}_{k} \in \mathbb{C}^{M_{k} \times d_{k}}$. The resultant signal at receiver $k$ is given by

$$
\begin{aligned}
\hat{\mathbf{x}}_{k} & =\mathbf{W}_{k}^{H} \mathbf{y}_{k} \\
& =\mathbf{W}_{k}^{H} \mathbf{H}_{k, k} \mathbf{F}_{k} \mathbf{x}_{k}+\sum_{\ell \in \mathcal{K} \backslash\{k\}} \mathbf{W}_{k}^{H} \mathbf{H}_{k, \ell} \mathbf{F}_{\ell} \mathbf{x}_{\ell}+\mathbf{W}_{k}^{H} \mathbf{z}_{k} .
\end{aligned}
$$

It can be observed from (3) that the received signal suffers inter-user interference not only from the secondary but also from the primary network. Different from the general interference channels in which all the users in the network cooperate to design their transmission strategies in order to optimize the system performance, the cooperation between PUs and SUs is in general limited in CR networks. In such, the design of the precoders $\mathbf{F}_{k}$ and receive filter matrices $\mathbf{W}_{k}$ to efficiently handle interference is a fundamental challenge.

To design the transmission strategies of the users, we adopt the CSI assumptions similar to [2], [18], [21-23]. The perfect CSI of the primary links and secondary links is assumed to be available at the SUs. In practice, the secondary receiver can estimate its associated CSI and then feedback to its transmitter. In addition, the SU can obtain the crosschannels to the primary receivers by sensing the signal transmitted from the primary receiver in time-division duplexing (TDD). Since the channels are assumed be fixed in the processing period, the cross-channel matrices can be exchanged in the secondary networks [2]. Furthermore, we assume the CSI is perfectly known at the users. For the cases in which the CSI is imperfectly known, the results in this paper are served as a benchmark [18], [21], [23].

To perfectly eliminate all interference in (3), a full IA scheme can be applied. Then, the precoders and receive matrices are designed to meet the following conditions [10], [11]:

$$
\begin{aligned}
& \mathbf{W}_{k}^{H} \mathbf{H}_{k, \ell} \mathbf{F}_{\ell}=\mathbf{0}, \quad k, \ell \in \mathcal{K}, k \neq \ell, \\
& \operatorname{rank}\left(\mathbf{W}_{k}^{H} \mathbf{H}_{k, k} \mathbf{F}_{k}\right)=d_{k}, \quad k \in \mathcal{K} .
\end{aligned}
$$

The transceivers satisfying the above conditions can be designed by an iterative algorithm using the interference leakage minimization in [10]. As discussed in [16], full IA can result in zero inter-user interference but the sum-rate performance of the PUs can be significantly degraded since the PUs sacrifice their own transmission rate to assist the SUs to achieve the interference-free transmission. In this paper, we will propose the Min-Max MSE based IA in which interference from the SUs to the PUs is completely eliminated while the SUs adapt their transmission strategies to improve the performance.

\section{Problem Formulation and Proposed Method}

In this section, we consider optimizing the precoding and receiver matrices to improve the worst user MSE performance of the system subject to individual transmitted power constraints. Since the PUs have higher priority to access the spectrum than the SUs, the PUs can selfishly design their transmission strategies without awareness of the presence of the SUs. On the other hand, the SU transmission strategies must guarantee no harmful interference to the PUs.

\subsection{Primary Transmission Strategies}

It is worth mentioning that the PUs typically offer limited cooperation with the SUs, the PUs determine their transmission strategies based on the knowledge of channels between the PUs [5]. From (3), the post-processed signal at PU $k, k \in \mathcal{K}_{p}$, can be rewritten as

$$
\begin{aligned}
\hat{\mathbf{x}}_{k}= & \underbrace{\mathbf{W}_{k}^{H} \mathbf{H}_{k, k} \mathbf{F}_{k} \mathbf{x}_{k}}_{\text {desired signal }}+\underbrace{\sum_{\ell \in \mathcal{K}_{p} \backslash\{k\}} \mathbf{W}_{k}^{H} \mathbf{H}_{k, \ell} \mathbf{F}_{\ell} \mathbf{x}_{\ell}}_{\text {interference from PUs }} \\
& +\underbrace{\sum_{\ell \in \mathcal{K}_{s}} \mathbf{W}_{k}^{H} \mathbf{H}_{k, \ell} \mathbf{F}_{\ell} \mathbf{x}_{\ell}}_{\text {interference from SUs }}+\underbrace{\mathbf{W}_{k}^{H} \mathbf{z}_{k}}_{\text {noise }}, \quad k \in \mathcal{K}_{p} .
\end{aligned}
$$

The SU transmission must not cause any interference to the PUs. Thus, the zero-interference conditions should be imposed.

$$
\mathbf{W}_{k}^{H} \mathbf{H}_{k, \ell} \mathbf{F}_{\ell}=\mathbf{0}, \ell \in \mathcal{K}_{s}, k \in \mathcal{K}_{p} .
$$

Accordingly, the MSE of PU $k, k \in \mathcal{K}_{p}$, from (6) is defined by

$$
\begin{aligned}
\xi_{k}\left(\mathbf{F}_{k}, \mathbf{W}_{k}\right) & =\mathbb{E}\left[\left\|\hat{\mathbf{x}}_{k}-\mathbf{x}_{k}\right\|_{2}^{2}\right] \\
& =\left\|\mathbf{I}-\mathbf{W}_{k}^{H} \mathbf{H}_{k, k} \mathbf{F}_{k}\right\|_{F}^{2}+\sigma_{k}^{2}\left\|\mathbf{W}_{k}\right\|_{F}^{2} \\
& +\sum_{\ell \in \mathcal{K}_{p} \backslash\{k\}}\left\|\mathbf{W}_{k}^{H} \mathbf{H}_{k, \ell} \mathbf{F}_{\ell}\right\|_{F}^{2}, \quad k \in \mathcal{K}_{p} .
\end{aligned}
$$

It can be seen that minimizing the MSE in (8) results in the interference leakage minimization in IA schemes [24]. 
Thus, the MSE minimization is also known as MSE-based IA [24], [25]. To provide fairness among users, we design the precoders and receive filters at PUs to minimize the worst user MSE among PUs. The design problem can be mathematically formulated as

$$
\begin{array}{cl}
\min _{\left\{\mathbf{W}_{k}, \mathbf{F}_{k}\right\}_{k \in \mathcal{K}_{p}}} & \max _{k \in \mathcal{K}_{p}} \xi_{k}\left(\mathbf{F}_{k}, \mathbf{W}_{k}\right), \\
\text { s.t. } & \left\|\mathbf{F}_{k}\right\|_{F}^{2} \leq P_{k, \max }, \quad k \in \mathcal{K}_{p} .
\end{array}
$$

It can be shown that the optimization problem (9) is not jointly convex in the precoder and receive matrices. In addition, the transceiver matrices $\left\{\mathbf{F}_{k}\right\}$ and $\left\{\mathbf{W}_{k}\right\}$ are coupled with each other in the objective function. Thus, it is difficult to optimize the transceivers simultaneously. Fortunately, the optimization problem (9) is convex for individual variables while the others are fixed. Thus, we adopt the alternating optimization algorithm to iteratively solve (9) [24], [25]. First, given fixed receive matrices, we design the precoders which can be reformulated as

$$
\begin{array}{cl}
\min _{\left\{\mathbf{F}_{k}\right\}_{k \in \mathcal{K}_{p}}} & \max _{k \in \mathcal{K}_{p}} \xi_{k}\left(\mathbf{F}_{k}, \mathbf{W}_{k}\right), \\
\text { s.t. } & \left\|\mathbf{F}_{k}\right\|_{F}^{2} \leq P_{k, \max }, \quad k \in \mathcal{K}_{p} .
\end{array}
$$

By introducing an auxiliary variable $\eta_{p}$, the optimization problem (10) can be equivalently rewritten as

$$
\begin{array}{cl}
\min _{\left\{\mathbf{F}_{k}\right\}_{k \in \mathcal{K}_{p}}, \eta_{p}} & \eta_{p}, \\
& \sqrt{\xi_{k}\left(\mathbf{F}_{k}, \mathbf{W}_{k}\right)} \leq \eta_{p}, \quad k \in \mathcal{K}_{p}, \\
\text { s.t. } & \left\|\mathbf{F}_{k}\right\|_{F}^{2} \leq P_{k, \max }, \quad k \in \mathcal{K}_{p} .
\end{array}
$$

Next, we shall reformulate the optimization problem (11) into a tractable one. Note that $\xi_{k}\left(\mathbf{F}_{k}, \mathbf{W}_{k}\right)$ can be equivalently rewritten as

$\xi_{k}\left(\mathbf{F}_{k}, \mathbf{W}_{k}\right)=\left\|\mathbf{W}_{k}^{H} \mathbf{\Phi}_{k} \mathbf{H}_{p} \mathbf{F}_{p}-\Delta_{k}\right\|_{F}^{2}+\sigma_{k}^{2}\left\|\mathbf{W}_{k}\right\|_{F}^{2}, \quad k \in \mathcal{K}_{p}$

where we have defined

$$
\begin{gathered}
\mathbf{H}_{p}=\left[\begin{array}{cccc}
\mathbf{H}_{1,1} & \mathbf{H}_{1,2} & \cdots & \mathbf{H}_{1, K_{p}} \\
\mathbf{H}_{2,1} & \mathbf{H}_{2,2} & \cdots & \mathbf{H}_{2, K_{p}} \\
\vdots & \vdots & \vdots & \vdots \\
\mathbf{H}_{K_{p}, 1} & \mathbf{H}_{K_{p}, 2} & \cdots & \mathbf{H}_{K_{p}, K_{p}}
\end{array}\right], \\
\boldsymbol{\Phi}_{k}=\left[\begin{array}{lll}
\mathbf{0}_{M_{k} \times \sum_{\ell=1}^{k-1} M_{\ell}} & \mathbf{I}_{M_{k}} & \mathbf{0}_{M_{k} \times \sum_{\ell=k+1}^{K_{p}} M_{\ell}}
\end{array}\right], \\
\boldsymbol{\Delta}_{k}=\left[\begin{array}{lll}
\mathbf{0}_{d_{k} \times \sum_{\ell=1}^{k-1} d_{\ell}} & \mathbf{I}_{d_{k}} & \mathbf{0}_{d_{k} \times \sum_{\ell=k+1}^{K p} d_{\ell}}^{K p}
\end{array}\right],
\end{gathered}
$$

and

$$
\mathbf{F}_{p}=\operatorname{blkdiag}\left(\mathbf{F}_{1}, \mathbf{F}_{2}, \cdots, \mathbf{F}_{K_{p}}\right)
$$

Applying formulas $\|\mathbf{X}\|_{F}^{2}=(\operatorname{vec}(\mathbf{X}))^{H} \operatorname{vec}(\mathbf{X})=$ $\|\operatorname{vec}(\mathbf{X})\|_{2}^{2}$ and $\operatorname{vec}(\mathbf{A B C})=(\mathbf{I} \otimes \mathbf{A B}) \operatorname{vec}(\mathbf{C})$ into (12), one has

$$
\begin{aligned}
\xi_{k}\left(\mathbf{F}_{k}, \mathbf{W}_{k}\right)= & \left\|\left[\mathbf{I}_{d_{p}} \otimes\left(\mathbf{W}_{k}^{H} \mathbf{\Phi}_{k} \mathbf{H}_{p}\right)\right] \operatorname{vec}\left(F_{p}\right)-\operatorname{vec}\left(\boldsymbol{\Delta}_{k}\right)\right\|_{2}^{2}, \\
& +\sigma_{k}^{2}\left\|\mathbf{W}_{k}\right\|_{F}^{2} \\
& =\left\|\begin{array}{c}
\delta_{k} \\
\boldsymbol{\Gamma}_{k} \operatorname{vec}\left(F_{p}\right)-\operatorname{vec}\left(\Delta_{k}\right)
\end{array}\right\|_{2}^{2}
\end{aligned}
$$

with $d_{p}=\sum_{k \in \mathcal{K}_{p}} d_{k}, \delta_{k}=\sigma_{k}\left\|\mathbf{W}_{k}\right\|_{F}, \quad \boldsymbol{\Gamma}_{k}=\mathbf{I}_{d_{p}} \otimes$ $\left(\mathbf{W}_{k}^{H} \boldsymbol{\Phi}_{k} \mathbf{H}_{p}\right)$, for $k \in \mathcal{K}_{p}$. The precoder design in (11) becomes

$$
\begin{gathered}
\min _{\left\{\mathbf{F}_{k}\right\}_{k \in \mathcal{K}_{p}}, \eta_{p}} \eta_{p}, \\
\delta_{k} \\
\text { s.t. }\left\|\boldsymbol{\Gamma}_{k} \operatorname{vec}\left(F_{p}\right)-\operatorname{vec}\left(\Delta_{k}\right)\right\|_{2} \leq \eta_{p}, k \in \mathcal{K}_{p}, \\
\left\|\operatorname{vec}\left(\mathbf{F}_{k}\right)\right\|_{2} \leq \sqrt{P_{k, \max }} k \in \mathcal{K}_{p} .
\end{gathered}
$$

It can be observed from (14) that the objective is linear while the constraints are second-order cones. Thus, the optimization problem (14) is known as SOCP which can be efficiently solved by available solvers such as SeDuMi, CVX [27], [28].

Next, by fixing the precoders, we design the receive filter matrices. Note that the receive filter matrix at one PU only affects its own MSE. Thus, the receive matrix design for the PRx $k, k \in \mathcal{K}_{p}$, from (9) is recast as

$$
\min _{\mathbf{W}_{k}} \xi_{k}\left(\mathbf{F}_{k}, \mathbf{W}_{k}\right) \text {. }
$$

Taking the partial derivative of $\xi_{k}\left(\mathbf{F}_{k}, \mathbf{W}_{k}\right)$ with respect to $\mathbf{W}_{k}^{\dagger}$ and setting it to zero, one can obtain the optimal receive filter as

$$
\mathbf{W}_{k}=\left[\sum_{\ell \in \mathcal{K}_{p}} \mathbf{H}_{k, \ell} \mathbf{F}_{\ell} \mathbf{F}_{\ell}^{H} \mathbf{H}_{k, \ell}^{H}+\sigma_{k}^{2} \mathbf{I}\right]^{-1} \mathbf{H}_{k, k} \mathbf{F}_{k}, k \in \mathcal{K}_{p} .
$$

Denote the objective function in (9) as $\psi_{p}\left(\mathbf{F}_{k}, \mathbf{W}_{k}\right)=$ $\max _{k \in \mathcal{K}_{p}} \xi_{k}\left(\mathbf{F}_{k}, \mathbf{W}_{k}\right)$. $\epsilon$ denotes an acceptable error tolerance. The iterative algorithm for the design of the PU precoding and receive matrices is summarized in Algorithm 1.

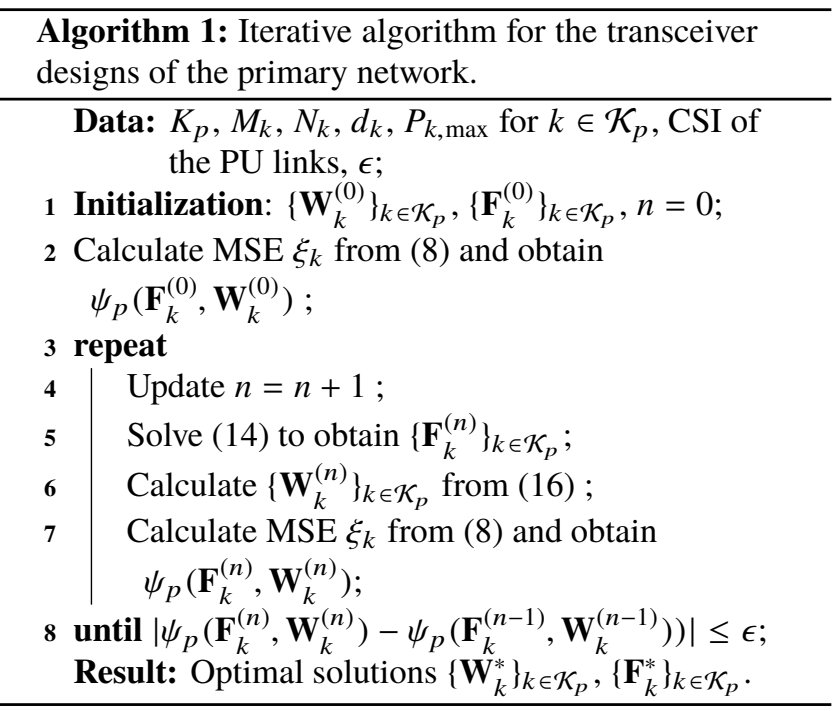

Note that to design the precoders and receive filters at the PUs, the PUs are oblivious of the presence of the SUs 
and do not need to know the CSI related to the SUs. The PUs require only the CSI of the PU links. Let $\mathbf{F}_{k}^{(n)}$ and $\mathbf{W}_{k}^{(n)}$ be the solutions to (14) and (15) at iteration $n$, respectively. Since the optimization problem (14) minimizes the maximum MSE while the receive filters are fixed, we have $\psi_{p}\left(\mathbf{F}_{k}^{(n)}, \mathbf{W}_{k}^{(n)}\right) \geq \psi_{p}\left(\mathbf{F}_{k}^{(n+1)}, \mathbf{W}_{k}^{(n)}\right)$. Similarly, the maximum MSE is minimized with the receiver filter in (16), i.e., $\psi_{p}\left(\mathbf{F}_{k}^{(n+1)}, \mathbf{W}_{k}^{(n)}\right) \geq \psi_{p}\left(\mathbf{F}_{k}^{(n+1)}, \mathbf{W}_{k}^{(n+1)}\right)$. Thus, one has $\psi_{p}\left(\mathbf{F}_{k}^{(n)}, \mathbf{W}_{k}^{(n)}\right) \geq \psi_{p}\left(\mathbf{F}_{k}^{(n+1)}, \mathbf{W}_{k}^{(n)}\right) \geq \psi_{p}\left(\mathbf{F}_{k}^{(n+1)}, \mathbf{W}_{k}^{(n+1)}\right)$.

This means that the worst user MSE is monotonically reduced at each iteration. In addition, the MSE is lower bounded by zero. Thus, Algorithm 1 is convergent at least to a local optimal solution.

With regard to computational complexity, the major computational complexity of Algorithm 1 comes from solving the SOCP (14) to find $\left\{\mathbf{F}_{k}^{(n)}\right\}_{k \in \mathcal{K}_{p}}$ and calculating $\left\{\mathbf{W}_{k}^{(n)}\right\}_{k \in \mathcal{K}_{p}}$ from (16). The SOCP is solved with the computational complexity of $O\left(\left(\sum_{k \in \mathcal{K}_{p}} N_{k} d_{k}\right)^{2}\left(2 K_{p}\right)^{2.5}+\right.$ $\left.\left(2 K_{p}\right)^{3.5}\right)$ [28] while the computational complexity of finding $\left\{\mathbf{W}_{k}^{(n)}\right\}_{k \in \mathcal{K}_{p}}$ is $\mathcal{O}\left(\sum_{k \in \mathcal{K}_{p}} M_{k}^{3}\right)$. Thus, the overall computational complexity of Algorithm 1 is approximated by $O\left(\left(\sum_{k \in \mathcal{K}_{p}} N_{k} d_{k}\right)^{2}\left(2 K_{p}\right)^{2.5}+\left(2 K_{p}\right)^{3.5}+\sum_{k \in \mathcal{K}_{p}} M_{k}^{3}\right)$ for each iteration.

\subsection{Secondary Transmission Strategies}

Given the PU transmission strategies, the SUs adapt their transmission to optimize their transmission performance while not creating harmful interference to the PUs. From (3), the estimated signal at SU $k, k \in \mathcal{K}_{s}$, can be rewritten as

$$
\begin{aligned}
\hat{\mathbf{x}}_{k}= & \underbrace{\mathbf{W}_{k}^{H} \mathbf{H}_{k, k} \mathbf{F}_{k} \mathbf{x}_{k}}_{\text {desired signal }}+\underbrace{\sum_{\ell \in \mathcal{K}_{s} \backslash\{k\}} \mathbf{W}_{k}^{H} \mathbf{H}_{k, \ell} \mathbf{F}_{\ell} \mathbf{x}_{\ell}}_{\text {interference from SUs }} \\
& +\underbrace{\sum_{\ell \in \mathcal{K}_{p}} \mathbf{W}_{k}^{H} \mathbf{H}_{k, \ell} \mathbf{F}_{\ell} \mathbf{x}_{\ell}}_{\text {interference from PUs }}+\underbrace{\mathbf{W}_{k}^{H} \mathbf{z}_{k}}_{\text {noise }}, \quad k \in \mathcal{K}_{s} .
\end{aligned}
$$

Different from the PUs, the SUs must cope with interference from both SUs and PUs. To enhance the system performance, the SUs exploit the CSI of the channel links from the SUs to PUs and between the SUs to design the optimal transceivers. From (18), the MSE for SU $k$ is given by

$$
\begin{gathered}
\xi_{k}\left(\mathbf{F}_{k}, \mathbf{W}_{k}\right)=\mathbb{E}\left[\left\|\hat{\mathbf{x}}_{k}-\mathbf{x}_{k}\right\|_{2}^{2}\right], \\
=\left\|\mathbf{I}-\mathbf{W}_{k}^{H} \mathbf{H}_{k, k} \mathbf{F}_{k}\right\|_{F}^{2}+\sum_{\ell \in \mathcal{K}_{s} \backslash\{k\}}\left\|\mathbf{W}_{k}^{H} \mathbf{H}_{k, \ell} \mathbf{F}_{\ell}\right\|_{F}^{2} \\
+\sum_{\ell \in \mathcal{K}_{p}}\left\|\mathbf{W}_{k}^{H} \mathbf{H}_{k, \ell} \mathbf{F}_{\ell}\right\|_{F}^{2}+\sigma_{k}^{2}\left\|\mathbf{W}_{k}\right\|_{F}^{2}, \quad k \in \mathcal{K}_{s} .
\end{gathered}
$$

With the $\mathcal{K}_{p_{0}}$ ditions of zero-interference from the SUs to the PUs given in (7), the fairness Min-Max MSE transceiver design for the SUs is expressed as

$$
\begin{array}{cl}
\min _{\left\{\mathbf{W}_{k}, \mathbf{F}_{k}\right\}_{k \in \mathcal{K}_{s}}} & \max _{k \in \mathcal{K}_{s}} \xi_{k}\left(\mathbf{F}_{k}, \mathbf{W}_{k}\right), \\
\text { s.t. } & \left\|\mathbf{F}_{k}\right\|_{F}^{2} \leq P_{k, \max }, \quad k \in \mathcal{K}_{s}, \\
& \mathbf{W}_{k}^{H} \mathbf{H}_{k, \ell} \mathbf{F}_{\ell}=\mathbf{0}, k \in \mathcal{K}_{p}, \ell \in \mathcal{K}_{s} .
\end{array}
$$

Note that condition (20c) guarantees that interference from the SUs does not spill into the desired signal subspace at the PU receivers. To satisfy condition (20c), the SU precoder, $\mathbf{F}_{\ell}, \ell \in \mathcal{K}_{s}$ must lie in the null space of $\mathbf{W}_{k}^{H} \mathbf{H}_{k, \ell}$ for all $k \in \mathcal{K}_{p}$. It implies that

$\mathbf{F}_{\ell} \in \mathcal{N}\left(\mathbf{W}_{1}^{H} \mathbf{H}_{1, \ell}\right) \cap \mathcal{N}\left(\mathbf{W}_{2}^{H} \mathbf{H}_{2, \ell}\right) \cap \ldots \cap \mathcal{N}\left(\mathbf{W}_{K_{p}}^{H} \mathbf{H}_{K_{p}, \ell}\right)$.

By defining matrix $\mathcal{H}_{\ell} \in \mathbb{C}^{\left(\sum_{k \in \mathcal{K}_{p}} d_{k}\right) \times M_{\ell}}$ as

$$
\mathcal{H}_{\ell}=\left[\left(\mathbf{W}_{1}^{H} \mathbf{H}_{1, \ell}\right)^{H},\left(\mathbf{W}_{2}^{H} \mathbf{H}_{2, \ell}\right)^{H}, \ldots,\left(\mathbf{W}_{K_{p}}^{H} \mathbf{H}_{K+p, \ell}\right)^{H}\right]^{H},
$$

equation (21) is rewritten as

$$
\mathbf{F}_{\ell} \in \mathcal{N}\left(\mathcal{H}_{\ell}\right)
$$

Then, the necessary condition for existence of nullity of matrix $\mathcal{H}_{\ell}$ is $M_{\ell} \geq \sum_{k \in \mathcal{K}_{p}} d_{k}$. Accordingly, a null space of $\mathcal{H}_{\ell}$ can be found by [26]

$$
\boldsymbol{\Psi}_{\ell}=\left[\mathbf{I}_{M_{\ell}}-\mathcal{H}_{\ell}^{H}\left(\mathcal{H}_{\ell} \mathcal{H}_{\ell}^{H}\right)^{-1} \mathcal{H}_{\ell}\right]_{1}^{M_{\ell}-\sum_{k \in \mathcal{K}_{p}} d_{k}} .
$$

Then, to fulfill condition (23), we propose to design each precoder as a product of two matrices given by

$$
\mathbf{F}_{\ell}=\Psi_{\ell} \tilde{\mathbf{F}}_{\ell}
$$

where $\tilde{\mathbf{F}}_{\ell} \in \mathbb{C}^{\left(M_{\ell}-\sum_{k \in \mathcal{K}_{p}} d_{k}\right) \times d_{\ell}}$ is designed to improve the performance of the SUs. It should be noted that with the structure in (25), the signals transmitted from the SUs are not pilled into the desired signal subspace at the PUs. Them, the optimization problem (20) can be rewritten as

$$
\begin{array}{cl}
\min _{\left\{\tilde{\mathbf{F}}_{k}, \mathbf{W}_{k}\right\}_{k \in \mathcal{K}_{s}}} & \max _{k \in \mathcal{K}_{s}} \xi_{k}\left(\tilde{\mathbf{F}}_{k}, \mathbf{W}_{k}\right), \\
\text { s.t. } & \left\|\boldsymbol{\Psi}_{k} \tilde{\mathbf{F}}_{k}\right\|_{F}^{2} \leq P_{k, \max }, k \in \mathcal{K}_{s} .
\end{array}
$$

Similar to the PU transceiver designs, the optimization problem (26) is nonconvex with respect to the design variables. However, it is convex with respect to the precoder matrix variables when the receive filter matrices are fixed, and vice versa. Thus, we employ the alternating optimization. By fixing the receive filters, the precoder design can be represented as

$$
\begin{array}{cl}
\min _{\left\{\tilde{\mathbf{F}}_{k}\right\}_{k \in \mathcal{K}_{s}}} & \max _{k \in \mathcal{K}_{s}} \xi_{k}\left(\tilde{\mathbf{F}}_{k}, \mathbf{W}_{k}\right), \\
\text { s.t. } & \left\|\boldsymbol{\Psi}_{k} \tilde{\mathbf{F}}_{k}\right\|_{F}^{2} \leq P_{k, \max }, k \in \mathcal{K}_{s} .
\end{array}
$$

To transform problem (27) into a tractable convex optimization, we define

$$
\begin{gathered}
\mathbf{H}_{s}=\left[\begin{array}{cccc}
\mathbf{H}_{K_{p}+1, K_{p}+1} & \mathbf{H}_{K_{p}+1, K_{p}+2} & \cdots & \mathbf{H}_{K_{p}+1, K} \\
\mathbf{H}_{K_{p}+2, K_{p}+1} & \mathbf{H}_{K_{p}+2, K_{p}+2} & \cdots & \mathbf{H}_{K_{p}+2, K} \\
\vdots & \vdots & \vdots & \vdots \\
\mathbf{H}_{K, K_{p}+1} & \mathbf{H}_{K, K_{p}+2} & \cdots & \mathbf{H}_{K, K}
\end{array}\right] \\
\boldsymbol{\Psi}_{s}=\operatorname{blkdiag}\left(\boldsymbol{\Psi}_{K_{p}+1}, \boldsymbol{\Psi}_{K_{p}+1}, \cdots, \boldsymbol{\Psi}_{K_{p}+1}\right)
\end{gathered}
$$




$$
\begin{gathered}
\boldsymbol{\Phi}_{k}=\left[\begin{array}{lll}
\mathbf{0}_{M_{k} \times \sum_{\ell=K p+1}^{k-1} M_{\ell}} & \mathbf{I}_{M_{k}} & \mathbf{0}_{M_{k} \times \sum_{\ell=k+1}^{K} M_{\ell}}^{K}
\end{array}\right], \\
\boldsymbol{\Delta}_{k}=\left[\begin{array}{lll}
\mathbf{0}_{d_{k} \times \sum_{\ell=K p+1}^{k-1} d_{\ell}} & \mathbf{I}_{d_{k}} & \mathbf{0}_{d_{k} \times \sum_{\ell=k+1}^{K} d_{\ell}}^{K}
\end{array}\right],
\end{gathered}
$$

and

$$
\mathbf{F}_{s}=\operatorname{blkdiag}\left(\tilde{\mathbf{F}}_{K_{p}+1}, \tilde{\mathbf{F}}_{K_{p}+2}, \cdots, \tilde{\mathbf{F}}_{K}\right)
$$

Then, the MSE in (19) of the SUs can be rewritten as

$$
\begin{gathered}
\xi_{k}\left(\tilde{\mathbf{F}}_{k}, \mathbf{W}_{k}\right)=\left\|\mathbf{W}_{k}^{H} \mathbf{\Phi}_{k} \mathbf{H}_{s} \mathbf{\Psi}_{s} \mathbf{F}_{s}-\boldsymbol{\Delta}_{k}\right\|_{F}^{2} \\
+\sum_{\ell \in \mathcal{K}_{p}}\left\|\mathbf{W}_{k}^{H} \mathbf{H}_{k, \ell} \mathbf{F}_{\ell}\right\|_{F}^{2}+\sigma_{k}^{2}\left\|\mathbf{W}_{k}\right\|_{F}^{2}, k \in \mathcal{K}_{s},
\end{gathered}
$$

or, equivalently,

$$
\xi_{k}\left(\tilde{\mathbf{F}}_{k}, \mathbf{W}_{k}\right)=\left\|\begin{array}{c}
\delta_{k} \\
\boldsymbol{\Gamma}_{k} \operatorname{vec}\left(F_{s}\right)-\operatorname{vec}\left(\boldsymbol{\Delta}_{k}\right)
\end{array}\right\|_{2}^{2},
$$

where $\delta_{k}=\left(\sum_{\ell \in \mathcal{K}_{p}}\left\|\mathbf{W}_{k}^{H} \mathbf{H}_{k, \ell} \mathbf{F}_{\ell}\right\|_{F}^{2}+\sigma_{k}^{2}\left\|\mathbf{W}_{k}\right\|_{F}^{2}\right)^{1 / 2}$, and $\boldsymbol{\Gamma}_{k}=\mathbf{I}_{d_{s}} \otimes\left(\mathbf{W}_{k}^{H} \boldsymbol{\Phi}_{k} \mathbf{H}_{s} \boldsymbol{\Psi}_{s}\right)$ with $d_{s}=\sum_{k \in \mathcal{K}_{s}} d_{k}$. Then, the precoder design for the SUs is written as the following SOCP

$$
\begin{gathered}
\min _{\left\{\tilde{\mathbf{F}}_{k}\right\}_{k \in \mathcal{K}_{s}}, \eta_{s}} \eta_{s}, \\
\text { s.t. } \\
\delta_{k} \\
\quad\left\|\left(\mathbf{I}_{d_{k}} \otimes \boldsymbol{\Psi}_{k}\right) \operatorname{vec}\left(\tilde{\mathbf{F}}_{k}\right)\right\|_{2} \leq \sqrt{P_{k, \max }} \quad k \in \mathcal{K}_{s},
\end{gathered}
$$

where $\eta_{s}$ is an additional auxiliary variable. After achieving the optimal precoders, the next step is to design the receive filters. It can be noted that each receive filter of the user affects to its own MSE rather than the other users. With the obtained precoders from (30), we can express the design problem of the receive filter for the SRx $k$ as follows

$$
\min _{\mathbf{W}_{k}} \xi_{k}\left(\tilde{\mathbf{F}}_{k}, \mathbf{W}_{k}\right), \quad k \in \mathcal{K}_{s} .
$$

The optimal receive matrices can be obtained by solving $\partial \xi_{k} / \partial \mathbf{W}_{k}^{\dagger}=0$ which results in

$$
\mathbf{W}_{k}=\left[\sum_{\ell \in \mathcal{K}} \mathbf{H}_{k, \ell} \boldsymbol{\Psi}_{\ell} \tilde{\mathbf{F}}_{\ell} \tilde{\mathbf{F}}_{\ell}^{H} \boldsymbol{\Psi}_{\ell}^{H} \mathbf{H}_{k, \ell}+\sigma_{k}^{2} \mathbf{I}\right]^{-1} \mathbf{H}_{k, k} \boldsymbol{\Psi}_{k} \tilde{\mathbf{F}}_{k},
$$

The detailed alternating optimization for the SU transceiver designs is given in Algorithm 2 in which we have defined $\psi_{s}\left(\mathbf{F}_{k}, \mathbf{W}_{k}\right)=\max _{k \in \mathcal{K}_{s}} \xi_{k}\left(\mathbf{F}_{k}, \mathbf{W}_{k}\right)$. Note that from (19) the SUs need to estimate the total interference from the PUs to design their transceivers in the secondary network [6]. In addition, it is observed from (20c) that the SUs require the knowledge of the receive filters at the PUs. The SUs can obtain the knowledge of the PU receive filters by sensing the information exchanged from the PRx to the PTxs or by exploiting the central fusion center [22] or the spectrum manager [29].

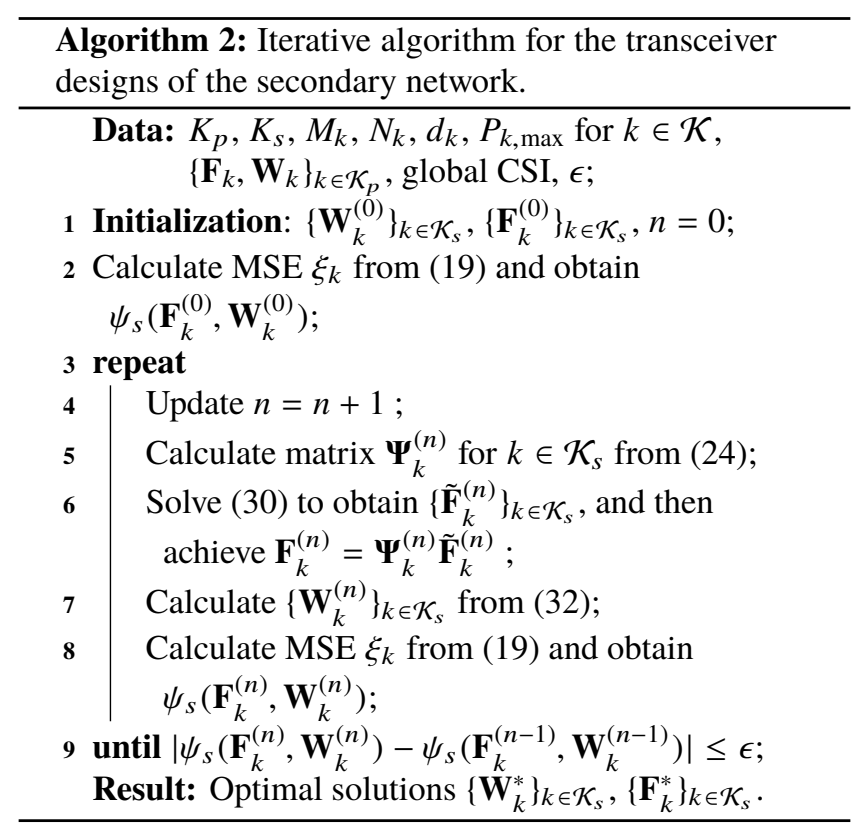

Similar to Algorithm 1, it is easy to show that Algorithm 2 is also guaranteed to be convergent at least to a local optimal solution since we have

$$
\psi_{s}\left(\mathbf{F}_{k}^{(n)}, \mathbf{W}_{k}^{(n)}\right) \geq \psi_{s}\left(\mathbf{F}_{k}^{(n+1)}, \mathbf{W}_{k}^{(n)}\right) \geq \psi_{s}\left(\mathbf{F}_{k}^{(n+1)}, \mathbf{W}_{k}^{(n+1)}\right) .
$$

The major computational complexity of Algorithm 2 to find $\left\{\mathbf{F}_{k}^{(n)}\right\}_{k \in \mathcal{K}_{s}}$ by calculating $\boldsymbol{\Psi}_{k}^{(n)}$ for $k \in \mathcal{K}_{s}$ from (24) and solving the SOCP (30) is $O\left(\left(\sum_{\ell \in K_{s}}\left(M_{\ell}-\right.\right.\right.$ $\left.\left.\left.\sum_{i \in \mathcal{K}_{p}} d_{i}\right) d_{\ell}\right)^{2}\left(2 K_{s}\right)^{2.5}+\left(2 K_{s}\right)^{3.5}+\left(\sum_{k \in \mathcal{K}_{p}} d_{k}\right)^{3}\right)$ [28]. The computational complexity of finding $\left\{\mathbf{W}_{k}^{(n)}\right\}_{k \in \mathcal{K}_{s}}$ is $O\left(\sum_{k \in \mathcal{K}_{s}} M_{k}^{3}\right)$. Thus, the overall computational complexity for each iteration in Algorithm 2 is about $\mathcal{O}\left(\left(\sum_{\ell \in \mathcal{K}_{s}}\left(M_{\ell}-\sum_{i \in \mathcal{K}_{p}} d_{i}\right) d_{\ell}\right)^{2}\left(2 K_{s}\right)^{2.5}+\left(2 K_{s}\right)^{3.5}+\right.$ $\left.\left(\sum_{k \in \mathcal{K}_{p}} d_{k}\right)^{3}+\sum_{k \in \mathcal{K}_{s}} M_{k}^{3}\right)$.

\section{Simulation Results}

In this section, we provide simulation results to validate the performance of the proposed Min-Max MSE based IA. In all simulations, the MIMO Rayleigh channel coefficients are randomly generated with zero mean and unit variance. We consider that $\sigma_{k}^{2}=\sigma_{n}^{2}=1, k \in \mathcal{K}, P_{k, \max }=P_{p \text {, max }}$ for $k \in \mathcal{K}_{p}$ and $P_{k, \text { max }}=P_{s, \text { max }}$ for $k \in \mathcal{K}_{s}$. We define the signal to noise ratio (SNR) of the PUs as $\gamma_{p}=\frac{P_{p, \max }}{\sigma_{n}^{2}}$ and the SNR of the SUs as $\gamma_{s}=\frac{P_{s, \max }}{\sigma_{n}^{2}}$. We consider the CR network with $K_{p}=2$ PUs and $K_{s}=2$ SUs. We set $N_{k}=6, M_{k}=2$, $d_{k}=2$ for $k \in \mathcal{K}$, and $\epsilon=10^{-6}$. We carry out Monte-Carlo simulations over 200 channel realizations.

First, we study the convergence characteristics of the proposed Min-Max MSE method. We set $\gamma_{p}=\gamma_{s}=10 \mathrm{~dB}$. We choose initial precoder and receive filter matrices to be 


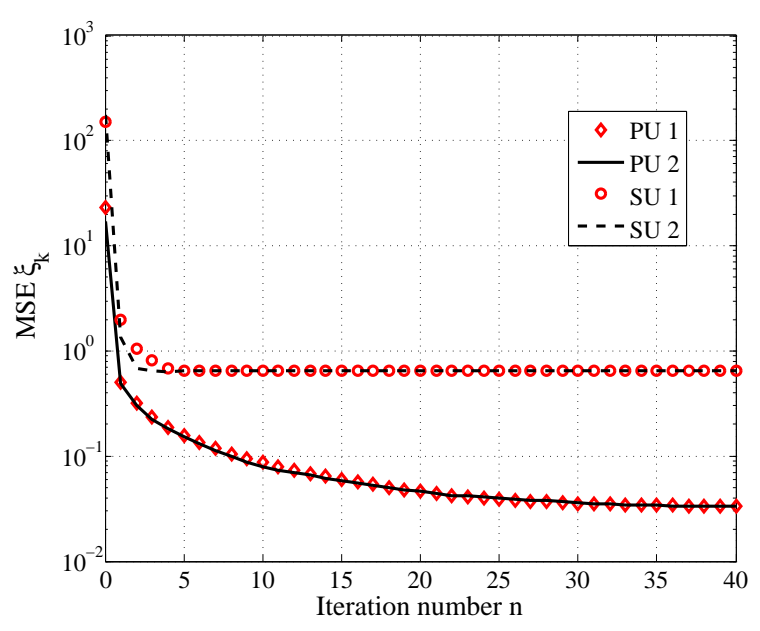

Fig. 2. Convergence of the proposed alternating optimization for the Min-Max MSE transceiver design problems.

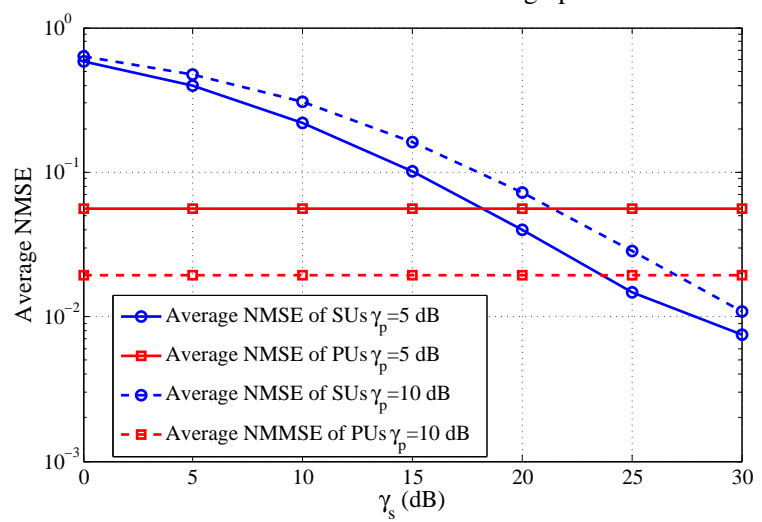

Fig. 3. Average NMSEs of the PUs and SUs versus the SU SNR.

right and left singular matrices of the corresponding desired signal links. The evolution of the user MSEs obtained from each iteration is plotted in Fig. 2 for a channel realization. As observed from the figure that the algorithm is convergent in less than 40 iterations. It should be noted that at the optimum, the MSEs of the SUs are the same. Similarly, the MSEs of all PUs are identical at the optimum. This confirms the MSE fairness among users in the same network. In addition, with the same SNR, the MSE performance of the PUs is better than that of the SUs. That is because the PUs have higher priority to access the spectrum while the SUs must sacrifice their performance to maintain no harmful interference to the PUs.

Next, we investigate the MSE performance of the proposed method in terms of the average MSE normalized by the number of the data streams (NMSE) [30] and we study the impact of the SU transmission on the performance of the PUs. Figure 3 displays the average worst-user NMSE versus the SU SNR for the primary and secondary networks with $\gamma_{p}=\{5,10\} \mathrm{dB}$. It can be seen from Fig. 3 that when the transmitted power of the PUs increases, i.e., $\gamma_{p}$ increases from $5 \mathrm{~dB}$ to $10 \mathrm{~dB}$, the MSE performance of the PUs is improved. On the other hand, an increase in the PU transmitted power results in the MSE performance degradation of the SUs. That is because that an increase in transmitted power of

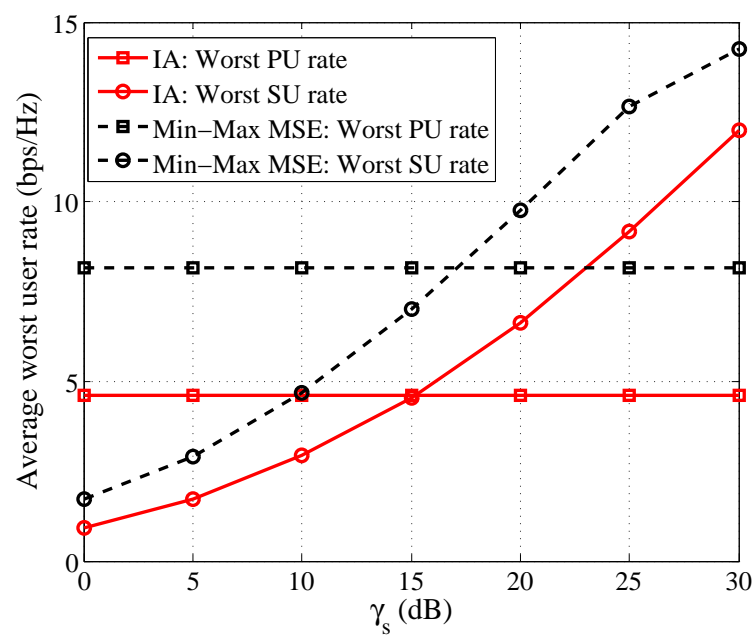

Fig. 4. Average worst user rates of the PUs and SUs versus the SU SNR for the proposed Min-Max MSE in comparison with those for IA in [17].

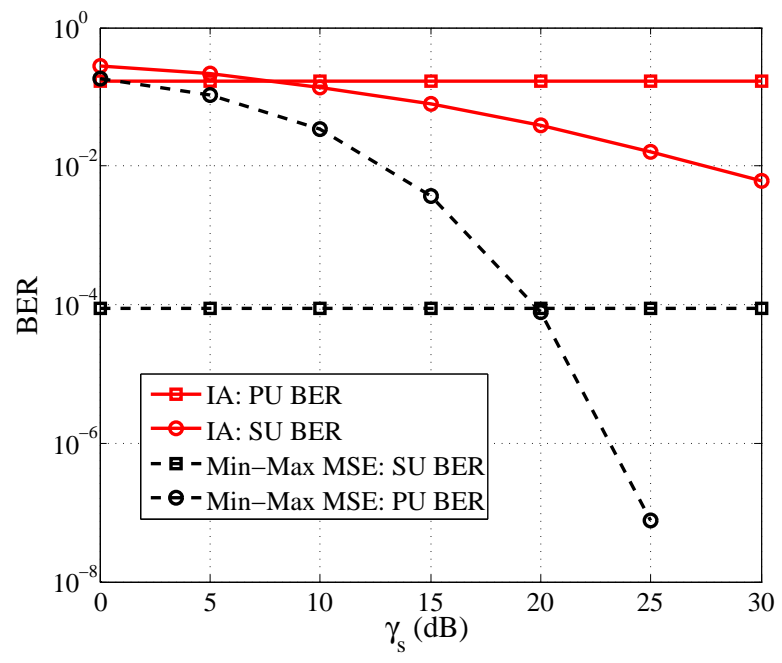

Fig. 5. BER of the PUs and SUs versus the SU SNR for the proposed Min-Max MSE in comparison with those for IA in [17].

the PUs causes severer interference to the SUs. In contrast, the MSEs of the PUs are unchanged while the transmitted power of the SUs increases. This confirms that the SUs do not cause harmful interference to the PUs.

Now, we compares the user information rate and bit eror rate (BER) performance of the proposed Min-Max MSE algorithm with the IA scheme in [17] in which the interference leakage minimization is adopted to find the transceivers. We plot the average worst-user information rate of the SUs and PUs versus the SU SNR in Fig. 4 for $\gamma_{p}=5 \mathrm{~dB}$. It can be observed from Fig. 4 that the proposed method outperforms the IA scheme in [17] in terms of the average worst user rate. The worst user rate of the primary network is unchanged when the SU SNR $\gamma_{s}$ increases. This result reveals that the PU performance is not affected by the transmission of the SUs. To investigate the BER performance, the transmitted signals are modulated by quadrature phase shift keying (QPSK) signal constellations. Figure 5 plots the average BER of all the users for the primary and secondary networks. It is clear 
from Fig. 5 that the proposed method offers the superior BER performance when compared to the IA scheme in [17].

\section{Conclusion}

This paper has presented the optimal designs of the transmission strategies for the multiuser MIMO CR network. Our approach is to adopt the Min-Max MSE performance metric as the objective function for the optimization problems. We have derived the SOCP problems for the precoder designs and the closed form solutions for the receive filter designs. We have introduced the structures of the precoders to guarantee that the SU transmission do not cause adverse interference to the PUs. The iterative algorithms for the transceiver designs are showed to be convergent in few tens of iteration and their computational complexity is low. The numerical results demonstrate that the proposed method provide MSE fairness among the users. They also show that the proposed method outperforms the previous IA approach in terms of the worst user rate and bit error rate.

\section{Acknowledgments}

This research is funded by Vietnam National Foundation for Science and Technology Development (NAFOSTED) under grant number 102.04-2013.46.

\section{References}

[1] WANG, B., LIU, K. R. Advances in cognitive radio networks: A survey. IEEE Journal of Selected Topics in Signal Processing, 2011, vol. 5, no. 1, p. 5-23. DOI: 10.1109/JSTSP.2010.2093210

[2] LIU, Y., DONG, L. Spectrum sharing in MIMO cognitive radio networks based on cooperative game theory. IEEE Transaction on Wireless Communications, 2014, vol. 13, no. 9, p. 4807-4820. DOI: 10.1109/TWC.2014.2331287

[3] GOLDSMITH, A., JAFAR, S. A., MARIC, I., et al. Breaking spectrum gridlock with cognitive radios: An information theoretic perspective. Proceedings of the IEEE, 2009, vol. 97, no. 5, p. 894-914. DOI: 10.1109/JPROC.2009.2015717

[4] HOSSEINI, S. A., ABOLHASSANI, B., SADOUGH, S. M. S. A new protocal for cooperative spectrum sharing in mobile cognitive radio networks. Radioengineering, 2015, vol. 24, no. 3, p. 757-764. DOI: $10.13164 /$ re.2015.0757

[5] DU, H., RATNARAJAH, T. Robust transceiver beamforming in MIMO cognitive radio via second-order cone programming. IEEE Transactions on Signal Processing, 2011, vol. 60, no. 2, p. 781-792. DOI: 10.1109/TSP.2011.2174790

[6] KIM, S. J., GIANNAKIS, G. B. Optimal resource allocation for MIMO ad hoc cognitive radio networks. IEEE Transactions on Information Theory, 2011, vol. 57, no. 5, p. 3117-3131. DOI: 10.1109/TIT.2011.2120270
[7] GUI, X., KANG, G. X., ZHANG, P. Sum-rate maximising in cognitive MIMO ad-hoc networks using weighted MMSE approach. Electronics Letters, 2012, vol. 48, no. 19, p. 1240-1242. DOI:10.1049/el.2012.1472

[8] ZHANG, Y., ANESE, E., GIANNAKIS, G. B. Distributed optimal beamformers for cognitive radios bobust to channel uncertainties. IEEE Transactions on Signal Processing, 2012, vol. 60, no. 12, p. 6495-6508. DOI: 10.1109/TSP.2012.2218240

[9] YAO, Y., LI, G., XU, J., et al. Space alignment based on regulaized inversion precoding in cognitive transmission. Radioengineering, 2015, vol. 24, no. 3, p. 824-829. DOI: 10.13164/re.2015.0824

[10] GOMADAM, K., CADAMBE, V. R., JAFAR, S. A. A distributed numerical approach to interference alignment and applications to wireless interference networks. IEEE Transactions on Information Theory, 2011, vol. 57, no. 6, p. 3309-3322. DOI: $10.1109 /$ TIT.2011.2142270

[11] CADAMBE, V. R., JAFAR, S. A. Interference alignment and degrees of freedom of the $K$-user interference channel. IEEE Transactions on Information Theory, 2008, vol. 54, no. 8, p. 3425-3441. DOI: 10.1109/TIT.2008.926344

[12] PAPAILIOPOULOS, D., DIMAKIS, A. Interference alignment as a rank constrained rank minimization. IEEE Transactions on Signal Processing, 2012, vol. 60, no. 8, p. 4278-4288. DOI: 10.1109/TSP.2012.2197393

[13] AlEXANDROPOUlOS, G. C., PAPADIAS, C. B. A reconfigurable iterative algorithm for the K-user MIMO interference channel. Signal Processing, 2013, vol. 93, no. 12, p. 353-3362. DOI: 10.1016/j.sigpro.2013.05.027

[14] MADDAH-ALI, M. A., MOTAHARI, A. S., KHANDANI, A K. Communication over MIMO X channels: Interference alignment, decomposition and performance analysis. IEEE Transactions on Information Theory, 2008, vol. 54, no. 8, p. 3457-3470. DOI: 10.1109/TIT.2008.926460

[15] CASTANHEIRA, D., SILVA, A., GAMEIRO, A. Set optimization for efficient interference alignment in heterogeneous networks. IEEE Transactions on Wireless Communications, 2014, vol. 13, no. 10, p. 5648-5660. DOI: 10.1109/TWC.2014.2322855

[16] MEN, H., ZHAO, N., JIN, M., et al. Optimal transceiver design for interference alignment based cognitive radio networks. IEEE Communications Letters, 2015, vol. 19, no. 8, p. 1442-1445. DOI: 10.1109/LCOMM.2015.2442243

[17] REZAEI, F., TADAION, A. Interference alignment in cognitive radio networks. IET Communications, 2014, vol. 8, no. 10, p. 1769-1777. DOI: 10.1049/iet-com.2013.0731

[18] PERLAZA, S. M., FAWAZ, N., LASAULCE, S., et al. From spectrum pooling to space pooling: Opportunistic interference alignment in MIMO cognitive networks. IEEE Transactions on Signal Processing, 2010, vol. 58, no. 7, p. 3728-3741. DOI: 10.1109/TSP.2010.2046084

[19] SHARMA, S. K., CHARZINOTAS, S., OTTERSTEN, B. Interference alignment for spectral coexistence of heterogeneous networks. EURASIP Journal on Wireless Communications and Networking, 2013, vol. 2013, no. 1, p. 1-14. DOI: 10.1186/1687-1499-2013-46

[20] ALI, S. S., CASTANHEIRA, D., SILVA, A., et al. Transmission cooperative strategies for MIMO-OFDM heterogeneous networks. Radioengineering , 2015, vol. 24, no. 2, p. 431-441. DOI: $10.13164 / \mathrm{re} .2015 .0431$

[21] ZHANG, R., LIANG, Y. C. Exploiting multi-antennas for opportunistic spectrum sharing in cognitive radio networks. IEEE Journal of Selected Topics in Signal Processing, 2008, vol. 2, no. 1, p. 88-102. DOI: 10.1109/JSTSP.2007.914894 
[22] MOSLEH, S., ABOUEI, J., AGHABOZORGI, M. R. Distributed opportunistic interference alignment using threshold-based beamforming in MIMO overlay cognitive radio. IEEE Transactions on Vehicular Technology, 2014, vol. 63, no. 8, p. 3783-3793. DOI: $10.1109 /$ TVT.2014.2305849

[23] KRIKIDIS, I. Space alignment for cognitive transmission in MIMO uplink channels. EURASIP Journal on Wireless Communications and Networking, 2010, vol. 2010, no. 1, p. 1-6. DOI: $10.1155 / 2010 / 465157$

[24] LU, E., MA, T., LU, I. T. Interference alignment-like behaviors of MMSE designs for general multiuser MIMO systems. In Proceedings of the IEEE Global Telecommunications Conference (GLOBECOM 2011). 2011, p. 1-5. DOI: 10.1109/GLOCOM.2011.6134284

[25] SHEN, H., LI, B., TAO, M., et al. MSE-based transceiver designs for the MIMO interference channel. IEEE Transaction on Wireless Communications, 2010, vol. 9, no. 11, p. 3480-3489. DOI: 10.1109/TWC.2010.091510.091836

[26] HORN, R. A., JOHNSON, C. R. Matrix Analysis. Cambridge University Press: 1986. ISBN: 0-521-30586-1

[27] GRANT, M., BOYD, S. CVX: MATLAB software for disciplined convex programming, version 2.1. Available at: http://cvxr.com/cvx

[28] PEAUCELle, D., HENRION, D., LABIT, Y., et al. Users guide for seumi interface 1.04, 2002. Available at: http://homepages.laas.fr/peaucell/software/sdmguide.pdf

[29] ISLAM, H., LIANG, Y. C., HOANG, A. T. Joint power control and beamforming for cognitive radio networks. IEEE Transactions on Wireless Communications, 2008, vol. 7, no. 7, p. 2415-2419. DOI: 10.1109/TWC.2008.061003

[30] KHANDAKER, M. R. A., RONG, Y. Transceiver optimization for multihop MIMO relay multicasting from multiple sources. IEEE Transaction on Wireless Communications, 2014, vol. 13, no. 9, p. 5162-5172. DOI: 10.1109/TWC.2014.2322361

\section{About the Authors...}

Ha Hoang KHA received the B.Eng. and M.Eng. degrees from Ho Chi Minh City University of Technology, in 2000 and 2003, respectively, and the Ph.D. degree from the University of New South Wales, Sydney, Australia, in 2009, all in Electrical Engineering and Telecommunications. From 2000 to 2004, he was a research and teaching assistant with the Department of Electrical and Electronics Engineering, Ho Chi Minh City University of Technology. He was a visiting research fellow at the School of Electrical Engineering and Telecommunications, the University of New South Wales, Australia, from 2009 to 2011. He was a postdoctoral research fellow at the Faculty of Engineering and Information Technology, University of Technology Sydney, Australia from 2011 to 2013. He is currently a lecturer at the Faculty of Electrical and Electronics Engineering, Ho Chi Minh City University of Technology, Vietnam. His research interests are in digital signal processing and wireless communications, with a recent emphasis on convex optimization techniques in signal processing for wireless communications.

Hung Quang TA received his B.Eng. and M.Eng. in Electronics and Telecommunications from the Hanoi University of Science and Technology (HUST), Hanoi, in 1996 and 2000, respectively. He received his PhD in Electrical Engineering \& Telecommunications from the University of New South Wales (UNSW), Australia, in 2012. Currently, he is a lecturer at Faculty of Information Technology, Hanoi University, Vietnam. His research interests include optimization techniques, signal processing, image processing, embedded systems and cryptography. 